\title{
Knuckling Down on Predictive Factors for Early Relapse after Posterolateral Percutaneous Endoscopic Lumbar Discectomy
}

Bibhudendu Mohapatra ${ }^{1,2}$, Nitin Adsul ${ }^{1}$, ${ }^{H}$ yeun Sung Kim${ }^{1}$, Byapak Paudel ${ }^{1}$, Jee-Soo Jang ${ }^{1}$, Jeong-Hoon Choi ${ }^{1}$, Sung Kyun Chung ${ }^{1}$, Jeong Hoon Kim', Il-Tae Jang ${ }^{3}$, Seong-Hoon $\mathrm{Oh}^{4}$

${ }^{1}$ Department of Neurosurgery, Nanoori Suwon Hospital, Suwon, Korea; ${ }^{2}$ Indian Spinal Injuries Center, New Delhi, India; ${ }^{3}$ Department of Neurosurgery, Nanoori Hospital, Seoul, Korea; ${ }^{4}$ Department of Neurosurgery, Nanoori Incheon Hospital, Incheon, Korea

Corresponding Author:

Hyeun-Sung Kim, MD, PhD

Department of Neurosurgery, Nanoori

Suwon Hospital, 295, Jungbu-daero,

Yeongtong-gu, Suwon 16503, Korea

Tel: +82-31-1688-9797

Fax: +82-31-8065-9701

E-mail: neuros@hanmail.net

Received: February 17, 2018

Revised: December 4, 2018

Accepted: December 26, 2018
Objective: Percutaneous endoscopic lumbar discectomy (PELD) has several advantages, but it is not used routinely due to early relapse and steep learning curve. We have studied the factors associated with early relapse in patients who underwent posterolateral PELD at or above the L4-5 level. Methods: In this retrospective study, we have enrolled 200 cases and divided them into 4 groups ( $, B, C$, and D) with 50 patients in each group, that had undergone PELD by 2 different techniques (inside-out and outside-in with or without anti-adhesive agent) and operated by 2 different surgeons between May 2009 and November 2010. The factors studied were - Age, gender, disc (degeneration grade, location, level), associated adjacent level herniated nucleus pulposus (HNP), episode (first or recurrent), anti-adhesive agent, annulus preservation, approach, disc height and segmental dynamic motion (discrepancy in flexion and extension). Statistical analysis was done by Pearson's chi-square test and $\mathrm{p}$ value (significance). The clinical results were evaluated by visual analogue scale (VAS). Results: The mean age and mean followup period was comparable in all four groups. The overall recurrence rate was 9.5\% (19/200). Average early relapse time was 3.26 months. Factors like Age of the patient, multilevel HNP and degeneration grade showed significant correlation with relapse rate. The change in VAS pre-operatively to post-operatively was significant across all groups $(p<0.001)$. Conclusion: Based on the results of this study, high grade disc degeneration, multilevel disc herniation, and early postoperative activity are significantly associated with early relapse after PELD.

Key Words: Endoscopic lumbar discectomy, Anti-adhesive agent, Recurrent disc herniation

\section{INTRODUCTION}

Since the time the first discectomy described by Mixter and Barr in 1934 ${ }^{1)}$, the surgical technique has evolved from open laminectomy to microdiscectomy to percutaneous endoscopic lumbar discectomy (PELD) for lumbar disc herniation. Despite the advancement in the techniques, the most common complication of a discectomy is a recurrence of herniation ${ }^{2)}$.

Percutaneous endoscopic spine surgery of the lumbar spine has many advantages, but it has two problems: a long learning curve and early recurrence of herniation. Endoscopic removal of the lumbar disc herniation has been divided into interlaminar and posterolateral, which have different discal preparation methods and extraction forces, resulting in different types of relapse patterns. The purpose of this study was to investigate factors associated with early relapse in patients who underwent postero- lateral percutaneous endoscopic lumbar discectomy (PELD) at or above the L4-5 level.

\section{MATERIAL AND METHODS}

\section{Patients}

In this retrospective study, 200 cases were included who have undergone PELD by three different techniques (Inside-out and Outside-in with or without an anti-adhesive agent) and operated by 2 different surgeons between May 2009 and November 2010 (Fig. 1). This study was performed in patients with a minimum follow-up between 6 months and 1 year. Patients whose radiculopathy settled after the operation and postoperative MRI showing complete removal of the herniated disc were included. Patient with MRI proved successful treatment after index surgery and recurrence of symptoms within the first 6 months with positive 
MRI findings are considered under early relapse. Percutaneous endoscopic lumbar discectomy is different from the open lumbar discectomy in the characteristics of recurrence. Because of that, the most important point is not a symptom-free interval, rather than, postoperative MRI confirmation and successful removal is more important.

Only disc herniations at L4-L5 or above were included, L5-S1 disc herniations were excluded as we prefer a percutaneous endoscopic interlaminar approach rather than the transforaminal approach in the L5-S1 HNP.

Table 1. Groups of patients with different surgical approaches and use of anti-adhesive agent

\begin{tabular}{cccc}
\hline \hline Group & Number & Approach & Anti-adhesive \\
\hline A & 50 & Inside out & + \\
B & 50 & Outside in & - \\
C & 50 & Inside out & - \\
D & 50 & Inside out & + \\
\hline
\end{tabular}

The patients were divided into 4 groups (Table 1): Group $(A, B, C$, and D) with 50 patients in each group. The group $A$, $B$ and $C$ patients were operated by surgeon 1 and group $D$ patients were operated by surgeon 2. The outside-in approach was used in Group B with the inside-out approach in rest 3 groups. Anti-adhesive agents were used in Group A and D only. We compared the difference in relapse rates between the two surgical approaches and between groups with and without antiadhesive agents use since there is no specific study in humans which explains its role in recurrence of disc prolapse ${ }^{3,4)}$.

The factors included in relation to Disc Relapse were divided into 4 categories (Table 2): Group 1 (Demographic factors) included - age and gender. Group 2 (Disc factors) included: disc degeneration scale, combined with herniated nucleus pulposus, and disc herniation event - primary or recurrent. Group 3 (Operation factors) included: anti-adhesive agent, the location of disc herniation, annulus preservation and approach. Group 4 (Segmental stability factors) included: disc height, disc level, and dynamic motion. Combined herniated nucleus pulpous refers to cases with or without multilevel disc herniation and degener-

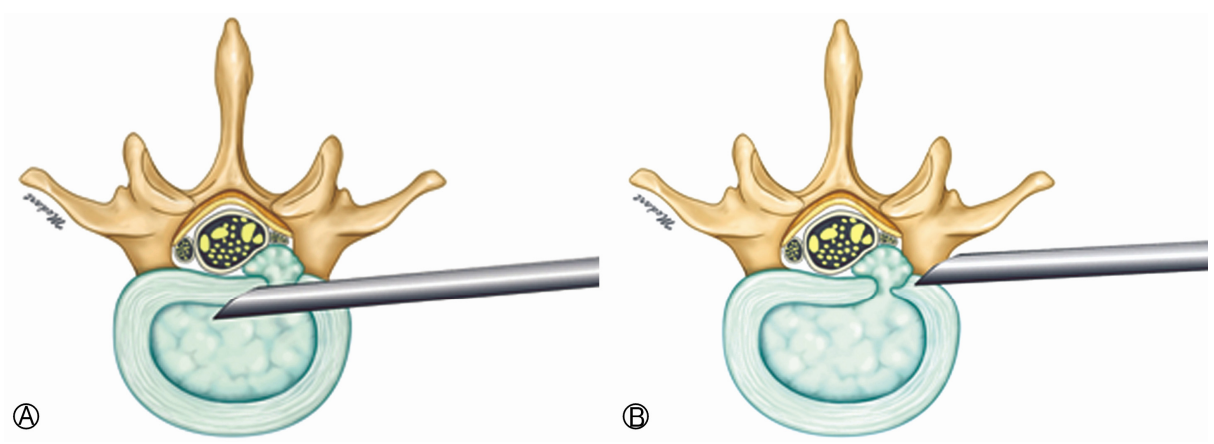

Fig. 1. (A) Inside out technique: working cannula docked inside the disc. (B) Outside in technique: working cannula docked to obtain half in and half out view of a disc.

Table 2. Factors considered in evaluating relapse rate after PELD

\begin{tabular}{|c|c|c|c|}
\hline Factors & Sub-Factors & Related 1 & Related 2 \\
\hline \multirow[t]{2}{*}{ Life Factors } & Age & & \\
\hline & Gender & & \\
\hline \multirow[t]{4}{*}{ Disc Factors } & Disc Degeneration Scale & DeCandido- scale 1-3 & \\
\hline & Combined HNP & 0 level to 3 levels & \\
\hline & Disc Herniation Event & First & \\
\hline & & Second & \\
\hline \multirow[t]{5}{*}{ Operation Factors } & Anti-Adhesive Agent & $+1-$ & \\
\hline & Disc Location & Central, Paracentral, Foraminal & \\
\hline & Annulus Preservation along the Disc Protrusion & & \\
\hline & Approach & Inside-Out & Group A, C, D \\
\hline & & Outside-In & Group B \\
\hline \multirow[t]{3}{*}{ Segmental Stability Factors } & Herniated Disc Level & & \\
\hline & Disc Height: Middle of Discal Space & $\frac{\text { HerniatedDisc }}{(\text { Upper }+ \text { Lower } / 2)}$ & \\
\hline & Segmental Dynamic Motion & Discrepancy of Flexion/Extension & \\
\hline
\end{tabular}


Table 3. Preoperative and postoperative VAS score

\begin{tabular}{cccccc}
\hline \hline Group & Follow-up (months) & Mean Age (years) & Recurrence (9.5\%-19/200) & VAS Pre-op & VAS Final follow up \\
\hline Group A & $21.12 \pm 4.57$ & $47.78 \pm 17.38$ & $12 \%(6 / 50)$ & $8.06 \pm 0.74$ & $1.44 \pm 0.54$ \\
Group B & $12.54 \pm 3.41$ & $45.52 \pm 14.40$ & $4 \%(2 / 50)$ & $8.34 \pm 0.63$ & $1.34 \pm 0.59$ \\
Group C & $19.00 \pm 4.42$ & $46.38 \pm 16.89$ & $6 \%(3 / 50)$ & $8.14 \pm 0.57$ & $1.86 \pm 0.86$ \\
Group D & $19.82 \pm 2.73$ & $47.16 \pm 15.64$ & $16 \%(8 / 50)$ & $8.10 \pm 0.65$ & $1.84 \pm 0.68$ \\
\hline
\end{tabular}

Table 4. Relation of age with recurrence

\begin{tabular}{ccccc}
\hline \hline Group (Yrs) & Patients (n) & Recurrence & $\%$ & p-value \\
\hline$<40$ & 79 & 3 & 3.79 & \\
$41-60$ & 78 & 14 & 17.9 & 0.003 \\
$>60$ & 43 & 2 & 4.6 & \\
Total & 200 & 19 & 9.5 & \\
\hline
\end{tabular}

ation but one was only symptomatic (graded 0 to 3 as per the number of discs involved apart from the index disc).

\section{Statistics}

The disc degeneration scale was graded as per DeCandido classification ${ }^{5)}$ which is based on loss of T2 hyperintensity in discs - Scale 1 (Normal or mild degeneration); Scale 2 (Moderate degeneration) and Scale 3 (Severely degenerated disc i.e. completely black disc). The disc height measurement was done using a midsagittal section of MRI (middle of the disc). The height is calculated by the formula - herniated level disc height divided by upper + lower disc height/2. The dynamic motion was measured using dynamic radiograph (flexion-extension). The visual analogue score (VAS) for pain was recorded pre- and post-operatively and at final follow up. Pearson's Chi-squared test (X2), Fisher's exact test and paired t-test were used to calculate p-value. Statistical software used for the study was SPSS version 20.0 (IBM Corp., Armonk, New York, USA).

\section{RESULTS}

The mean early relapse time was 3.26 months. Postoperative VAS was not significantly different between the four groups but a change in overall VAS in comparison to preoperative VAS was statistically significant $(p<0.001)$. The anti-adhesive group $(A$ and $D)$ had a higher rate of early recurrence than the untreated group ( $\mathrm{B}$ and $\mathrm{C}$ ). There was no significant difference in recurrence rate between the two groups, $B$, and $C$ i.e. between outside-in and inside-out approach (Table 3 ). Total relapse rate is 19 out of 200 . Out of which 13 patients treated conservatively, but six patients needed reoperation. Among them, four patients treated again revision endoscopic transforaminal lumbar discectomy. Only two cases required conversion to open microscopic lumbar discectomy.

Factors that showed correlation to early relapse were age, degeneration scale, combined HNP, anti-adhesive agent, disc location, disc level, and disc height but the statistically significant

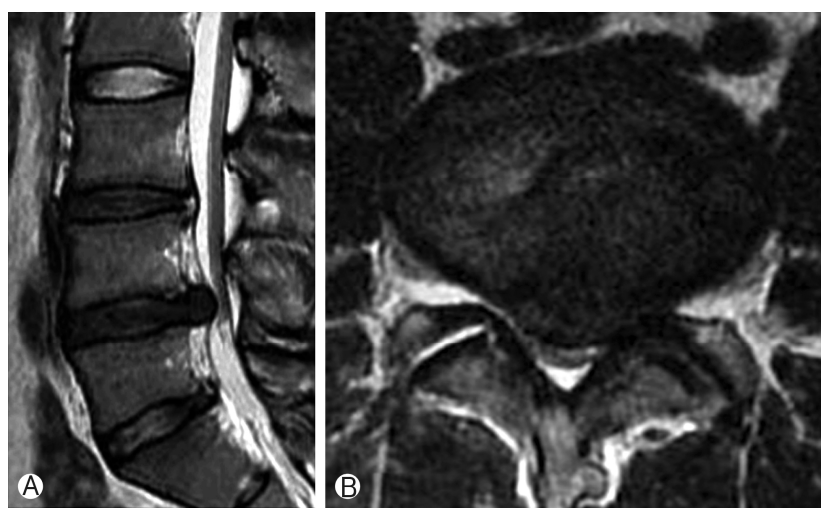

Fig. 2. (A), B) Pre-operative sagittal and axial MRI showing L4$\mathrm{L} 5$ disc herniation. Disc degeneration seen predominantly in the $44-5$ disc, rest of the discs are within normal limits.

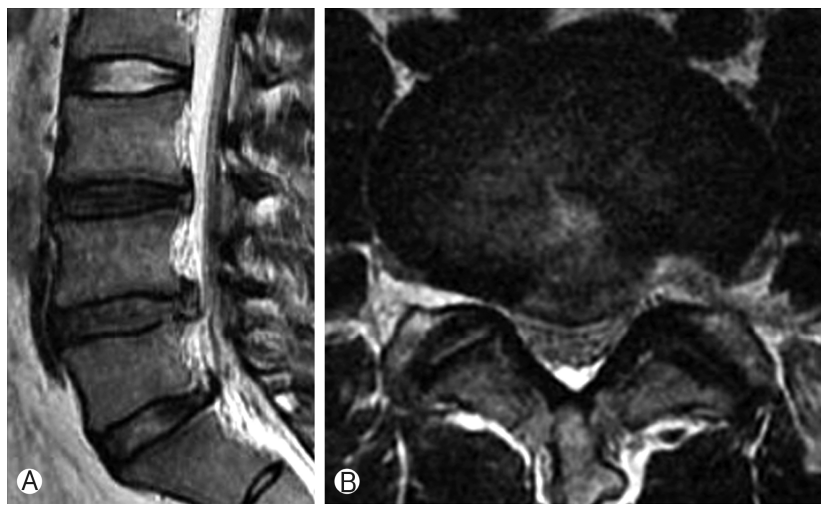

Fig. 3. (A), B) Immediate postoperative sagittal and axial MRI image after PELD.

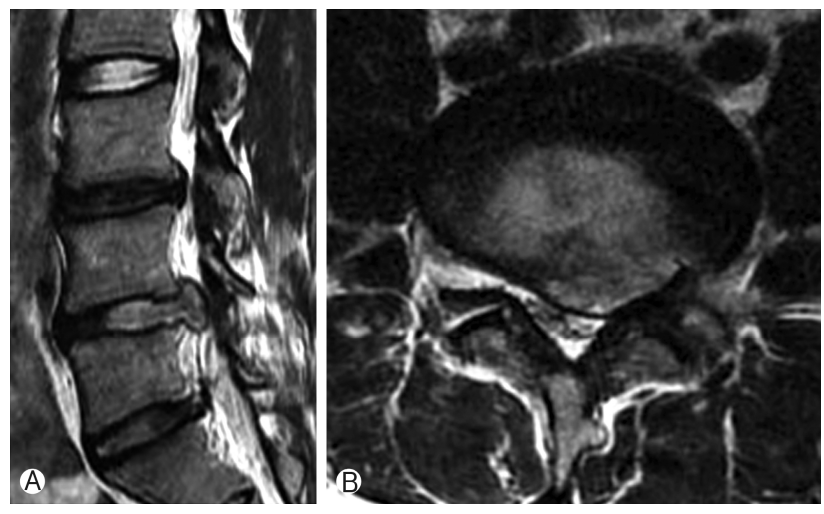

Fig. 4. (A), B) Recurrence of disc herniation after 3 months of PELD of the same patient as in figure 2 and 3, which operated with microscopic lumbar discectomy. 
correlation was seen only with age of the patient, combined HNP and degeneration grade.

As for each factor, the patients within the age group of

Table 5. The relation between degree of degeneration and recurrence

\begin{tabular}{ccccc}
\hline \hline Group & Patients $(\mathrm{n})$ & Recurrence & $\%$ & $\mathrm{p}$-value \\
\hline Scale 1 & 32 & 0 & 0 & \\
Scale 2 & 90 & 7 & 7.7 & 0.003 \\
Scale 3 & 78 & 12 & 15.3 & \\
Total & 200 & 19 & 9.5 & \\
\hline
\end{tabular}

Table 6. Relation of adjacent level HNP with recurrence

\begin{tabular}{ccccc}
\hline \hline Group & Patients $(\mathrm{n})$ & Recurrence & $\%$ & $p$-value \\
\hline 0 level & 85 & 3 & 3.5 & \\
1 level & 82 & 9 & 10.9 & \\
2 level & 25 & 5 & 20 & 0.001 \\
3 level & 8 & 2 & 25 & \\
Total & 200 & 19 & 9.5 & \\
\hline
\end{tabular}

40-60 years were most commonly affected. Hence with increasing age, the incidence of recurrence of herniation was greater $(p=0.003)$ (Table 4). There was no significant difference between male and female sex.

From our results, the relapse rate increased significantly with increase in the severity of degeneration $(p=0.003)$ (Table 5) i.e. more recurrence was seen in patients with grade/scale 3 degeneration as per DeCandido classification (Figs. 2, 3, 4). 32 patients showed scale 1 degeneration with no recurrence, 90 patients had scale 2 degeneration with recurrence in 7 patients. Scale 3 was seen in 78 patients with recurrence in 12 patients.

Patients with multiple HNP also showed significant correlation with early relapse following PELD $(p=0.001)$ (Table 6). However, there was no significant difference in relapse between patients with first-time herniation and second herniation (Fig. 5).

In the disc location, the relapse rate is relatively low in central, foraminal and far lateral in comparison to paracentral and migrated disc (superior and inferior) but the difference was not statistically significant. The most common level affected was an L4-5 disc (149/200) where 17 recurrences occurred. With increasing loss of disc height, there were more recurrences. Dynamic segmental instability along with disc height and disc

Table 7. Summary of the factors studied and its relation to recurrence

\begin{tabular}{|c|c|c|c|c|c|}
\hline \multirow{2}{*}{ FACTORS } & \multirow{2}{*}{ SUB-FACTORS } & \multirow{2}{*}{ RELATION } & \multicolumn{3}{|c|}{ Recurrence } \\
\hline & & & Level & Total & $\%$ \\
\hline \multirow{4}{*}{ Life Factor } & & & 40 years: & $3 / 79$ & $3.79 \%$ \\
\hline & Age & + & 41 60 years & $14 / 78$ & $17.9 \%$ \\
\hline & & & 61 years & $2 / 43$ & $4.6 \%$ \\
\hline & Sex & - & & & \\
\hline \multirow{8}{*}{ Disc Factor } & \multirow{3}{*}{ Degeneration Scale } & \multirow{3}{*}{+} & Scale 1 & $0 / 32$ & $0 \%$ \\
\hline & & & Scale 2 & $7 / 90$ & $7.7 \%$ \\
\hline & & & Scale 3 & $12 / 78$ & $15.3 \%$ \\
\hline & \multirow{4}{*}{ Combined HNP } & \multirow{4}{*}{+} & 0 Level & $3 / 85$ & $3.5 \%$ \\
\hline & & & 1 Level & 9/82 & $10.9 \%$ \\
\hline & & & 2 Level & $5 / 25$ & $20 \%$ \\
\hline & & & 3 Level & $2 / 8$ & $25 \%$ \\
\hline & Disc type (1st/recurrence) & - & & & \\
\hline \multirow{7}{*}{ Operation Factor } & \multirow{3}{*}{ Anti-adhesive agent } & \multirow{3}{*}{+} & Use & $14 / 100$ & $14 \%$ \\
\hline & & & No Use & $5 / 100$ & $5 \%$ \\
\hline & & & Central & $1 / 18$ & $5.56 \%$ \\
\hline & \multirow[t]{2}{*}{ Disc location } & \multirow[t]{2}{*}{+} & Foraminal/Far Lateral & $2 / 44$ & $4.5 \%$ \\
\hline & & & Inferior/Superior/Paracentral & $16 / 138$ & $11.59 \%$ \\
\hline & Annulus Preservation & - & & & \\
\hline & Approach & - & & & \\
\hline \multirow{6}{*}{ Segmental Stability Factor } & \multirow{3}{*}{ Disc level } & \multirow{3}{*}{+} & L1-2/L2-3/L3-4 & 2/51 & $3.92 \%$ \\
\hline & & & $\llcorner 4-5$ & $17 / 149$ & $11.41 \%$ \\
\hline & & & $\sim 80 \%$ & $4 / 23$ & $17.4 \%$ \\
\hline & \multirow[t]{2}{*}{ Disc height } & \multirow[t]{2}{*}{+} & $81 \sim 100 \%$ & 8/98 & $8.2 \%$ \\
\hline & & & $101 \% \sim$ & $7 / 79$ & $8.8 \%$ \\
\hline & Dynamic motion & - & & & \\
\hline
\end{tabular}




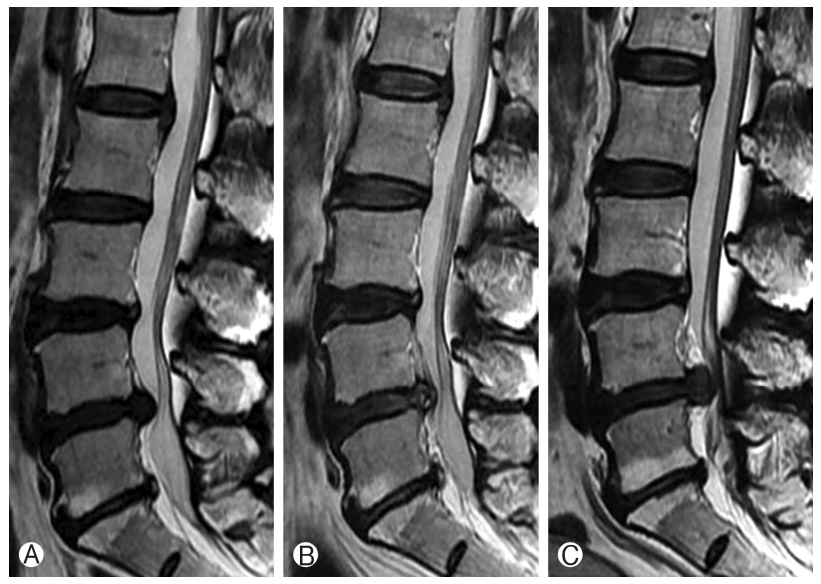

Fig. 5. (A) Sagittal MRI image of the lumbar spine shows multiple degenerated discs with a significant disc herniation at the L4-5 level. (B) The Immediate postoperative sagittal image of the lumbar spine of the same patient showing disc fragment removal with adequate decompression. (C) Sagittal MRI image of the same patient showing early recurrence at the onemonth postoperative period.

level did not show statistically significant difference regarding recurrence of herniation. Table 7 summarizes the factors studied and its correlation with relapse of disc herniation following PELD.

\section{DISCUSSION}

The recurrence rate after microsurgical lumbar discectomy ranges from $5-15 \%$ in various studies ${ }^{6,7}$. Regarding the definition of relapse or recurrence Swartz and Trost have described reherniation as the experience of another lumbar disc herniation more than 6 months after the index surgery, at either ipsilateral or contralateral side ${ }^{7)}$. But ideally, herniation at the same level and same side would be more appropriate for recurrence. However, it is not necessary to confine the time without pain to six months. If the symptom returns after a pain-free period even if earlier than 6 months, it can be defined as recurrence ${ }^{2)}$.

Jensdottir $\mathrm{M}$ et al. reported a recurrence rate of $12.7 \%$ occurring 10-20 years after microsurgical discectomies. The mean time to recurrence was 5.9 years (1.3-19.7 years $)^{8)}$. Carragee et al in their prospective study of 187 patients undergoing discectomies had a recurrence rate of $9 \%{ }^{9}$.

As per $\mathrm{Li} X$ et al. where they compared PELD with a standard discectomy, there were no significant differences in VAS, MacNab criteria at the final follow up, complications, recurrence rate, and re-operation rate ${ }^{10)}$. Ruetten et al. described a similar recurrent rate between microsurgical and endoscopic discectomy $(5.7 \% \text { Vs } 6.6 \%)^{11)}$. However, the reoperation risk was not higher after PELD for patients younger than 57 years, from 1.9 years, than open discectomy (Hazard ratio at $2 \mathrm{yr}, 0.86 ; 3 \mathrm{yr}, 0.78 ; 4$ $y r, 0.70 ;$ and $5 y r, 0.63)^{12,13)}$. The cumulative reoperation rate after 5 years was $13.4 \%$ and half of the reoperations occurred during the first postoperative year. With the exception of laminectomy, the reoperation rates of the other procedures were not different from that of open discectomy ${ }^{14)}$. In our study, the minimum relapse period was 3.26 months and the recurrence rate was $9.5 \%$ which well within the range reported in the literature. In our study, we checked successful surgery using the postoperative MRI for all patient. It may be related to the higher rate of early recurrence and early checking the recurrence of patient symptom and recurred disc herniation. As per Li $X$ et al. recurrence after open lumbar discectomy occurred lately. But, in the percutaneous endoscopic lumbar discectomy, recurrence occurred more early time rather than the late time. It may confuse the recurrence rate of percutaneous endoscopic lumbar discectomy more higher than the open lumbar discectomy. But, in our result, recurrence after percutaneous endoscopic lumbar discectomy occurred more early time. The probable cause for the early relapse after PELD may be the early return to normal activities before the annulus has healed completely and loading to the operated segment. Therefore, in order to prevent early relapse, healing of the annulus should be improved by minimizing segmental loading by applying a brace and sufficient restriction of activities of daily living in the postoperative period. This indicates that segmental motion has a great influence on recovery.

Many studies have described the risk factors for recurrence or relapse following open or microsurgical discectomy. Various estimated risk factors have been reported in the literature, such as age, gender, body mass index (BMI), smoking, herniation type, diabetes, and herniation level ${ }^{15-21)}$. However, the results of these studies were not always consistent. Regarding gender, Kim et al. reported that male gender was a risk factor for recurrence ${ }^{21)}$. However, many other observational studies did not find significant correlations between a male gender and recurrence ${ }^{16-18)}$. As for smoking, some studies revealed that smoking increased the risk of postoperative recurrence ${ }^{15,17,20)}$, whereas other studies found no significant difference between smokers and nonsmokers $^{14,18,20)}$. Huang et al. in their meta-analysis and systemic review of risk factors for recurrent lumbar disc herniation have concluded that smoking, diabetes and disc protrusion (contained) were predictors for recurrence ${ }^{15}$. In our study, we did not study smoking and diabetes as it was already an established factor.

There are very few studies on risk factors for recurrence after PELD. To our knowledge, this is the first study to evaluate the risk factors like the role of anti-adhesive agent ${ }^{3,4}$, comparisons of approach and associated HNP for relapse after PELD. As per $Y a o$ $Y$ et al. Obesity (BMI $\geq 25)$, age ( $\geq 50$ years old), the learning curve of the surgeon ( $<200$ cases), the treatment period and a central location of herniation were also closely associated with recurrent herniation after successful PELD ${ }^{16)}$. Kim JM et al. concluded that recurrent group had a higher mean age, higher body mass index, and the presence of protrusion or modic change increases recurrence after PELD ${ }^{17)}$. Kyoung-Tae Kim et al. demonstrated the strong correlation of disc height index, degeneration and sagittal range of motion with recurrence the following microdiscectomy ${ }^{18)}$. In our study increasing age was a statistically significant risk factor for relapse as shown 
in other studies. Degeneration of disc and multiple HNP strongly correlated with recurrence as evident in the literature. Regarding the location of disc central, foramina and far lateral disc were associated with less recurrence in comparison to paracentral and migrated disc although not significant. The factors disc height and segmental dynamic motion also did not correlate with recurrence. The reason may be in open discectomy, as described in most of the studies, the disc extraction force is from ventral to dorsal during the disc removal but in PELD the extraction force is from central to lateral.

Both the surgeons in our study have been doing endoscopic surgery for more than 10 years, so surgeons experience is less likely to affect the outcome. Several surgical procedures and treatments have been introduced to prevent recurrence after open lumbar discectomy, and sequestrectomy has been reported to be effective. Barth $\mathrm{M}$ et al. concluded that sequestrectomy has demonstrated significantly less postoperative disc degeneration than standard microdiscectomy after 2 years and may thus represent an attractive treatment alternative ${ }^{19)}$. Schick $U$ et al. found that Sequestrectomy does not seem to entail a higher rate of recurrences. The results are as favorable as or better than results after discectomy ${ }^{20)}$. Chiang $\mathrm{CJ}$ et al. in a porcine spine model showed modified purse-string suture provide effectively sealing for damaged annulus to withstand stresses $^{21)}$. Direct repair of annular incision has significantly slowed down degenerative process within discs after discectomy ${ }^{22)}$. In our study, 5 recurrences were seen out of 58 patients with annulus preservation (8.62\%) and 14 recurrences in 142 patients with annulus resected $(9.86 \%)$. The difference was not statistically significant. In the percutaneous endoscopic lumbar discectomy, many surgeons expect to preserve the complete annulus, which has the advantage of preserving the normal motion segment without causing adhesions in the operative field. However, it is associated with the higher chance of incomplete surgery. In our results, the recurrence rate of annulus preservation and annulus resection after successful removal of the pathologic disc was not statistically significant. It suggests that if feasible completely remove the pathologic disc, in spite of annulus resection. However long-term follow up may give more insight into the progression of disc degeneration.

\section{CONCLUSION}

In conclusion, the age, disc degeneration scale, and multilevel HNP are significant risk factors for early relapse. Chances of early recurrence after successful discectomy is common after PELD compared to the open lumbar discectomy. We should counsel the patient about early relapse in presence of high-grade disc degeneration at multiple levels. Moreover, absolute bed rest after percutaneous endoscopic lumbar discectomy for a few days will help in decreasing the early recurrence rate.

\section{ACKNOWLEDGEMENT}

We would like to acknowledge scientific team members Ms.
Jae Eun Park and Ms. Sol Lee for providing assistance in acquiring full-text articles and managing digital works.

\section{REFERENCES}

1. Mixter WJ: Rupture of the intertebral disc with involvement of the spinal canal. New Engl J Med 211:210-215, 1934

2. Shin B-J: Risk factors for recurrent lumbar disc herniations. Asian Spine Journal 8(2):211-215, 2014

3. Omlor G, Nerlich A, Lorenz H, Bruckner T, Richter W, Pfeiffer $\mathrm{M}$, et al: Injection of a polymerized hyaluronic acid/collagen hydrogel matrix in an in vivo porcine disc degeneration model. European Spine Journal 21(9):1700-1708, 2012

4. Guterl CC, See EY, Blanquer SB, Pandit A, Ferguson SJ, Benneker LM, et al: Challenges and strategies in the repair of ruptured annulus fibrosus. European Cells \& Materials 25:1, 2013

5. DeCandido P, Reinig JW, Dwyer AJ, Thompson KJ, Ducker TB: Magnetic resonance assessment of the distribution of lumbar spine disc degenerative changes. Journal of Spinal Disorders 1(1):9-15, 1988

6. McGirt MJ, Ambrossi GLG, Datoo G, Sciubba DM, Witham TF, Wolinsky J-P, et al: Recurrent disc herniation and long-term back pain after primary lumbar discectomy: review of outcomes reported for limited versus aggressive disc removal. Neurosurgery 64(2):338-345, 2009

7. Swartz KR, Trost GR: Recurrent lumbar disc herniation. Neurosurgical Focus 15(3):1-4, 2003

8. Jensdottir M, Gudmundsson K, Hannesson B, Gudmundsson G: 20 years follow-up after the first microsurgical lumbar discectomies in Iceland. Acta Neurochirurgica 149(1):51-58, 2007

9. Carragee EJ, Han MY, Suen PW, Kim D: Clinical outcomes after lumbar discectomy for sciatica: the effects of fragment type and anular competence. JBJS 85(1):102-108, 2003

10. Li X, Han Y, Di Z, Cui J, Pan J, Yang M, et al: Percutaneous endoscopic lumbar discectomy for lumbar disc herniation. Journal of Clinical Neuroscience 33:19-27, 2016

11. Ruetten S, Komp M, Merk H, Godolias G: Full-endoscopic interlaminar and transforaminal lumbar discectomy versus conventional microsurgical technique: a prospective, randomized, controlled study. Spine 33(9):931-939, 2008

12. Kim CH, Chung CK, Choi Y, Shin S, Kim MJ, Lee J, et al: The selection of open or percutaneous endoscopic lumbar discectomy according to an age cut-off point: nationwide cohort study. Spine 40(19):E1063-E1070, 2015

13. Kim K-T, Lee D-H, Cho D-C, Sung J-K, Kim Y-B: Preoperative Risk Factors for Recurrent Lumbar Disk Herniation in L5-S1. Clinical Spine Surgery 28(10):E571-E577, 2015

14. Kara B, Tulum Z, Acar Ü: Functional results and the risk factors of reoperations after lumbar disc surgery. European Spine Journal 14(1):43-48, 2005

15. Huang W, Han Z, Liu J, Yu L, Yu X: Risk factors for recurrent lumbar disc herniation: a systematic review and meta-analysis. Medicine 95(2), 2016

16. Yao Y, Liu $\mathrm{H}$, Zhang $\mathrm{H}$, Wang $\mathrm{H}$, Zhang $\mathrm{C}$, Zhang Z, et al: Risk factors for recurrent herniation after percutaneous endoscopic lumbar discectomy. World Neurosurgery 100:1-6, 2017

17. Kim J, Lee S, Ahn Y, Yoon D, Lee C, Lim S: Recurrence after 
successful percutaneous endoscopic lumbar discectomy. minMinimally Invasive Neurosurgery 50(02):82-85, 2007

18. Kim K-T, Park S-W, Kim Y-B: Disc height and segmental motion as risk factors for recurrent lumbar disc herniation. Spine 34(24): 2674-2678, 2009

19. Barth M, Diepers M, Weiss C, Thomé C: Two-year outcome after lumbar microdiscectomy versus microscopic sequestrectomy: part 2: radiographic evaluation and correlation with clinical outcome. Spine 33(3):273-279, 2008

20. Schick U, Elhabony R: Prospective comparative study of lumbar sequestrectomy and microdiscectomy. min-Minimally Invasive Neurosurgery 52(04):180-185, 2009

21. Chiang C-J, Cheng C-K, Sun J-S, Liao C-J, Wang Y-H, Tsuang Y-H: The effect of a new anular repair after discectomy in intervertebral disc degeneration: an experimental study using a porcine spine model. Spine 36(10):761-769, 2011

22. Kim HS, Park JY: Comparative assessment of different percuta neous endoscopic interlaminar lumbar discectomy (PEID) techniques. Pain Physician 16(4):359-367, 2013 